\title{
L'écriture « entre-les-langues » des auteures maghrébines de langue française et des auteures de «l'entre-des »
}

\author{
Roswitha Geyss \\ Département des langues romanes \\ Université de Vienne, Autriche
}

Dans cet article, nous retracerons le chemin des auteures maghrébines de langue française (Assia Djebar, Malika Mokeddem, Nine Moati) et des auteures de « l'entredeux » (Leïla Sebbar, Hélé Béji) dont nous examinerons l'œuvre, de leur entrée à l'école primaire à leur entrée en littérature. Il s'agit d'un chemin long et cahoteux, mais pourtant, on peut y déceler différentes étapes qui serviront de titre aux grandes parties de cet article : la tradition et la règle de la séparation des sexes; l'école et l'université ; l'écriture salvatrice, l'écriture de résistance et la résistance de l'écriture ; le travail ; et, sur le plan affectif, l'amour paternel et conjugal.

\section{La tradition et la règle de la séparation des sexes}

Écrire, si l'on est une femme de sensibilité arabo- ou berbéro-musulmane et d'éducation française, comme Assia Djebar et Malika Mokeddem, ou une femme de sensibilité juive méditerranéenne, perpétuant à la fois les idéaux du père socialiste et résistant, et de la mère forte et courageuse, comme Nine Moati, ou si l'on est le fruit d'un amour rapprochant les deux rives et suturant ce qui a été séparé par l'Histoire, comme Leïla Sebbar et Hélé Béji, est toujours un véritable acte de bravoure. La femme maghrébine, de sensibilité arabo- ou berbéro-musulmane, ou de sensibilité juive, quand elle s'empare de la parole publique, brise successivement tous les tabous qui pèsent sur son corps, sur son regard et sur sa voix. L'écriture demande toujours un mouvement du corps, ne serait-ce que le mouvement de la main qui trace les lettres, et la femme qui écrit, conquiert à petits pas l'espace public et masculin. Elle libère son regard, car on ne peut écrire sans regarder attentivement les autres et le monde. Et puis, sa voix devient mobile, les mots transcrits pouvant désormais franchir tous les obstacles. Monique Gadant constate que "Si elle s'empare de l'écrit, elle s'emparera de la parole, et menacera la règle de la séparation des sexes (infiçal), condition d'existence de la société. Elle violera la Loi que les hommes eux-mêmes doivent respecter. » (95) Concernant la division traditionnelle des espaces, Marta Segarra se rend compte que :

«Le père et la mère donc, en tant que personnages de ces romans [d'écrivaines maghrébines de langue française, R.G.], bien qu'ils soient valorisés de façons différentes et même opposées dépendant de chaque ouvrage, représentent presque toujours deux mondes distincts : celui du père, caractérisé par l'accès à l'espace extérieur et à une autre extériorité non moins im- 
portante fournie par l'éducation, normalement à travers la langue française ; et celui de la mère, représenté par l'intériorité de la maison familiale, les pulsions les plus primaires, la langue orale... En définitive, il s'agit des deux compartiments étanches réservés par la tradition aux hommes et aux femmes [nous soulignons]. » (106)

Monique Gadant parle de l'infiçal, de la «Loi » (avec une majuscule), qui est la « condition d'existence de la société » (95); Marta Segarra nomme l'instance qui édicte cette «Loi », que non seulement les femmes, mais aussi les hommes doivent respecter, la «tradition». Ce terme, par un effet métonymique, désigne aussi cette «Loi » elle-même. La tradition est donc une instance terrible aux multiples yeux et multiples oreilles qui écrase les individus devant se fondre dans l'anonymat du groupe et qui emprisonne les hommes autant que les femmes. Bien qu'elles transmettent depuis des millénaires les limites de l'univers féminin traditionnel à leurs filles, les femmes souffrent également de leur enfermement. Mais c'est aussi une «instance » qui offre une identité toute prête aux hommes et aux femmes, les dispensant ainsi de la fatigue mentale que cause l'obligation de s'inventer et de se réinventer sans cesse :

«Plus la société moderne se centre sur les individus, plus elle met au premier plan l'identité, ego cherchant à deviner et construire le sens de sa vie. Mais cette moderne énergie existentielle peine à trouver ses marques. Car les appartenances se font multiples, les ressources d'identification collective sont à inventer, ego est condamné à des bricolages incessants. Fatigué, il n'en est pas moins le centre obligé de ce qui donne sens à sa vie. À un stade moins avancé de l'individualisation au contraire (dans l'entre-deux entre régime communautaire et régime identitaire de l'individu), les débuts de la quête identitaire trouvent des ressources d'identification collective provenant de ce qu'il reste des structures communautaires. Il y a recyclage du communautaire par l'identitaire, produisant une identification collective qui peut entraîner et dominer les identités individuelles. » (Kaufmann 131-132)

Même aujourd'hui, fort nombreux et nombreuses sont les hommes et les femmes qui se replient sur les valeurs traditionnelles - ou ce que ceux-ci prennent pour telles, mais qui sont, en vérité, trop souvent des "anti-valeurs ", c'est-à-dire des "valeurs négatives » comme la haine et la violence, la tradition pouvant aussi être pervertie pour échapper à cette fatigue mentale : c'est ce que Kaufmann appelle la «contrerévolution identitaire » (Kaufmann 136) qui est un phénomène très moderne.

Mais la tradition, ce sont aussi ces valeurs qui ont permis à la population, notamment algérienne (la Tunisie et le Maroc étant des protectorats et gardant ainsi avec le bey à Tunis et le sultan à Rabat des dignitaires autochtones à la tête de l'Etat permettant à la population de se dire tunisienne ou marocaine) d'opposer aux arguments dits « culturels » du colonisateur, des arguments prouvant l'existence d'une Culture, la Culture algérienne, arabe, musulmane, mais aussi tamazight; d'une Langue, la Langue Arabe, langue du Coran, langue d'une tradition écrite millénaire; d'un Peuple, le Peuple algérien, et d'une Nation (Grandguillaume, « Les enjeux de la question des langues en Algérie »141). À cet égard, le sociologue Jean-Claude Kaufmann 
constate que les identités collectives se caractérisent par une "confirmation réciproque du sens de la vie » (142) et permettent ainsi de restaurer l'estime de soi.

Pendant l'époque coloniale, les valeurs traditionnelles (et sans aucun doute aussi les valeurs traditionnelles perverties devenues des " anti-valeurs ») sont entrées en contact avec tout ce qui fait partie de la modernité, tant celle de la " consommation » aussi bien qu'avec l'individualisme contemporain. En somme, on pourrait dire que la tradition et la modernité entrent en contact, tous les jours et partout, et que ce contact, ce « choc » produit quelque chose de nouveau, d'inouï. Nous sommes profondément persuadée que les tabous qui pèsent dans la culture " méditerranéenne » traditionnelle sur le corps, le regard et la voix de la femme, sont les mêmes qui, il n'y a pas encore si longtemps, pesaient aussi sur le corps, le regard et la voix de la femme dans les pays de l'hémisphère nord. Chaque pays suit son rythme de développement. Et ce n'est que par simple mépris (ou méprise) des faits historiques que les hommes de l'hémisphère nord se croient supérieurs à ceux de l'hémisphère sud, et $\mathrm{y}$ reviennent comme colonisateurs et usurpateurs, comme ce fut le cas en 1830 en Algérie, en 1881 en Tunisie et en 1912 au Maroc, et comme c'est le cas aujourd'hui en Iraq ou en Afghanistan. Au lieu de considérer la modernité et la tradition comme les deux pôles diamétralement opposés d'une échelle, nous sommes plutôt intéressée par l'échelle, c'est-à-dire par ce terrain fertile de «l'entre-deux». C'est cette zone périlleuse, mais aussi incroyablement fertile, que peuplent les auteures.

\section{L'école et l'université : la conversion du regard et l'askêsis}

C'est le père d'Assia Djebar qui conduit sa fille à l'école, un matin d'automne, pour qu'elle apprenne l'autre langue, qui lui permet de poursuivre ses études, même une fois l'âge nubile atteint, qui ne sera donc pas le geôlier, mais l'intercesseur, le libérateur. Cependant, malgré son enthousiasme juvénile, la jeune fille, qui aime tendrement sa mère voilée qui, seulement à l'âge de quarante ans et après l'installation de la famille à Alger, ose enfin enlever le voile et devient même mobile pour rendre visite à son fils, le prisonnier politique, en France, et qui aime tendrement ses tantes, ses cousines et ses grands-mères voilées - sa grand-mère paternelle si douce et humble, sa grand-mère maternelle forte et autoritaire -, s'aperçoit très vite de ses privilèges. «Adolescente ensuite, ivre quasiment de sentir la lumière sur ma peau, sur mon corps mobile, un doute se lève en moi : "Pourquoi moi ? Pourquoi à moi seule, dans la tribu, cette chance ?»(Djebar, L'Amour, la fantasia 297), se demande-elle, inquiète, émue. Il lui faudra plus d'un demi-siècle d'écriture (1956-2007) pour enlever, successivement, tous les voiles métaphoriques qui pèsent sur les femmes dans la culture arabo- ou berbéro-musulmane traditionnelle, pour dire enfin, sans fausse honte, « je », et pour dire enfin l'amour en français.

Les parents de Hélé Béji, sa mère chrétienne anticléricale et son père musulman libéral, veillent aussi avec grand soin à l'éducation de leur fille. Ils lui permettent de 
s'épanouir, et comme Assia Djebar, elle est pleinement consciente qu'elle doit tout à ses parents, qu'elle est une des rares filles privilégiées, ses parents l'ayant soustraite à l'emprise des gardiens et gardiennes de la tradition, des matrones sévères et cruelles (car ce sont surtout les femmes qui contribuent à maintenir intact le système patriarcal dont elles souffrent!). Hélé Béji en parle dans son essai Une force qui demeure (2006). Elle a la chance de se sentir aimée, et elle adore ses parents et sa grand-mère, à qui elle consacre son roman L'œil du jour (1985).

Malika Mokeddem, en revanche, doit livrer un combat exemplaire contre son père, sa mère et, enfin, contre tout le clan, pour pouvoir continuer ses études. Son père, un ouvrier pauvre, gardien d'un puits dans le désert, lui permet enfin de poursuivre ses études dans la ville voisine, grâce à l'intervention de son enseignante qui réussit à flatter son orgueil d'Algérien nationaliste: après tout, l'Algérie, une fois l'indépendance acquise, aura besoin de jeunes femmes instruites... Malika Mokeddem fait des études de médecine, malgré la pauvreté de sa famille, malgré l'hostilité croissante des «autres». Des hommes et des femmes, toujours vigilant(e)s, les hommes adossés au mur, les femmes très souvent cachées derrière les persiennes ou sous le voile, jusqu'à ce qu'elles ne laissent plus rien voir de leur visage qu'un seul œil au fond d'un tout petit orifice laissé ouvert, comme le font les «voyeuses » dans les fêtes de femmes depuis la nuit des temps, sont prêtes à désigner une jeune fille à la vindicte publique, uniquement parce qu'elle ose transgresser définitivement les limites de l'univers féminin. Malika Mokeddem est une de ces filles, parce qu'elle ose quitter sa famille pour s'installer seule en ville, parce qu'elle croise tous les jours dans la rue, dans les couloirs ou les cages d'escalier de la cité universitaire qui était encore mixte à cette époque-là, dans les amphithéâtres, des hommes, des étudiants comme elle.

Nine Moati est la fille de Serge Moati, d'un père fort et courageux, socialiste, francmaçon rattaché à la communauté des Granas, journaliste notamment à Tunis socialiste et au Petit Matin. Serge Moati se considère comme un Tunisien d'abord, comme un Tunisien surtout, il est mû par l'amour de son pays qu'il transmettra à ses enfants. Il rêve de participer à l'édification d'une société égalitaire et solidaire, il est convaincu qu'on peut aimer l'autre tout en restant fidèle à soi-même. Serge Moati est arrêté pendant la Seconde Guerre mondiale et déporté au camp de concentration de Sachsenhausen dont il s'echappera. Par la suite, il participe à la libération de Paris avant de retrouver sa famille. Nine Moati doit aussi beaucoup à sa mère Odette Scemama qui, elle, est issue de la communauté des Twânsa. Après l'arrestation de son mari, la mère de Nine se retrouve seule avec ses enfants dans la France vichyste. Devenue sans aucune préparation chef de famille, elle fait preuve d'un courage inouï pour faire vivre ses enfants et les sauver de la terreur, comme tant d'autres femmes pendant les deux guerres mondiales, malgré la peur qui l'asphyxie, elle la juive. Odette, comme son mari, meurt très tôt, en 1957. Trop tôt pour sa fille qui a la vive sensation de rester pourtant, malgré cet éloignement irrémédiable, toujours attachée à elle. 
Le père de Leỉla Sebbar, maître d'école, est mû, tout comme le père d'Assia Djebar, par de grands idéaux humanistes. Ainsi, il veut instruire les garçons « indigènes », il veut les aider à s'émanciper. Musulman libéral, marié à une Française chrétienne, mais anticléricale comme le père de Hélé Béji, il construit dans la maison d'école une « petite République française », une République idéale :

«La petite France des instituteurs laïques, mon père et ma mère, est dirigée de main de maître pour devenir intra-muros une République idéale où s'exercent, au nom de la justice, de l'égalité, de la fraternité, les lois de l'apprentissage scolaire dans les livres de la France, la langue de la France, la géographie et l'histoire de la France. Ma mère nous élève en petites filles de la République française, dans sa langue, dans ses livres, elle nous transmet un savoir universel, une langue unique. » (Sebbar, L'arabe comme un chant secret 42)

Leïla Sebbar aime son père qui n'a pas appris sa langue à lui, l'arabe dialectal de la région de Tlemcen, à ses quatre enfants pour les protéger des injustices de la situation coloniale, afin d'emprunter l'expression d'Albert Memmi, ou du système colonial, afin d'emprunter la terminologie consacrée de Jean-Paul Sartre, et, plus tard, aussi des horreurs de la guerre d'indépendance. Certes, elle souffre de cet éloignement irrémédiable ( « [...] j'étais une étrangère, la fille du frère préféré, oui, mais pas la fille de la cousine. » Sebbar, Mes Algéries en France 56), mais en même temps, elle comprend que l'exil n'est pas seulement le lieu de tous les manques, c'est aussi le lieu de tous les possibles :

«Quand je dis: «J'ai besoin d'être séparée de l'Algérie, j'ai besoin d'être séparée de la langue de mon père - pour écrire! -, je veux dire que c'est pour cette raison que je n'apprends pas l'arabe, et c'est pour cette raison, je pense, que je ne vais pas là où je veux profondément aller, en Algérie. J'ai le sentiment que si j'allais dans ces endroits-là, où je ne suis pas allée encore, je n'écrirais plus. Je ne suis pas sûre de ça ; mais... c'est ce que je crois. (longue pause) C'est l'absence, c'est l'exil qui me font écrire, et si l'absence, et si l'exil n'est plus là - si j'apprends la langue de mon père - j'ai plus besoin d'écrire. Quand je peux aller en Algérie comme je le désire, cela signifierait que je n'écrirais plus et que je n'ai plus rien à écrire - et à dire ! Ça, ça arrivera si je peux aller en Algérie suivant mon propre désir... et dans les lieux d'enfance. » (Geyss, «Interview avec Leïla Sebbar. Paris, le 27 septembre $2007 »)$

\subsection{Le retour vers soi}

Peut-être que pour les femmes de sensibilité arabo-musulmane ${ }^{1}$, il est encore plus important que leur entrée en écriture se fasse en langue française (et non pas en arabe classique, la langue du Coran). Cette langue n'est pas écrasée par le poids religieux ; elle n'est pas seulement la langue du colonisateur, mais avant tout la langue de

\footnotetext{
1 « J'écris donc, et en français, langue de l'ancien colonisateur, qui est devenue néanmoins et irréversiblement celle de ma pensée, tandis que je continue à aimer, à souffrir, également à prier (quand parfois je prie) en arabe, ma langue maternelle." (Djebar, "Idiome de l'exil et langue de l'irréductibilité »), a dit fort bien la romancière, historienne et cinéaste algérienne Assia Djebar.
} 
l'école, la langue de la littérature française, la langue de la philosophie ${ }^{2}$. Ce n'est que cette langue, ce «butin de guerre », comme disait Kateb Yacine, qui leur permet, pour le temps de la lecture, pour le temps de l'écriture au moins, de se soucier d'elles-mêmes, alors que, dans la culture arabo-musulmane traditionnelle, les hommes et femmes doivent se fondre dans l'anonymat du groupe, et tout ce qui touche à l'intimité ne doit pas être dit si ce n'est par le biais de métaphores très allusives: "La règle fondamentale est de ne pas parler de soi et de ne pas livrer les affaires du privé qui doivent rester secrètes. Je parle ici d'une règle qui s'impose à toute la société et par suite s'impose à la littérature, aux auteurs des deux sexes, a fortiori à une femme écrivain. » (Gadant 94)

Influencées par la philosophie occidentale, par la philosophie classique, les futures écrivaines apprennent à se soucier de leur soi, apprennent à se regarder, à se soigner, et appliquent donc le principe socratico-platonicien de l'epimeleia heautou. Foucault parle de la « conversion à soi » (Foucault L'Herméneutique du sujet 197-214). Il ne s'agit pas simplement d'une conversion du regard, mais d'un mouvement de l'être tout entier. Il est moins question de constituer le soi comme un objet d'analyse, de déchiffrement, de réflexion. Il s'agit bien d'avoir sous ses yeux le but : le soi, de mesurer la distance qui nous sépare de ce but, et d'avoir bien présent à l'esprit ce qu'on peut faire pour l'atteindre (213-214) Bien d'années après, Assia Djebar se rend compte combien l'écriture (autobiographique) l'a aidée à retourner vers le soi :

«Oui, le projet autobiographique vous installe, tel un pharaon dans votre propre vie : tel un monarque absolu prévoyant un scribe pour ses derniers instants d'hôpital, de souffrance, d'agonie, jusqu'à la dernière parole - «scribe, écris pour ma main défaillante! Ainsi, jusqu'au bout j'aurais écrit ma vie!»

Dans cette perspective de mégalomane, le désir autobiographique, quand il s'étale, qu'il s'organise, que la main de l'auteur relayée par celle du scribe qu'on a payé pour suivre, pas à pas, les mots et les gestes de l'auteur finissant de vivre, ce projet devient vivre pour écrire! » (Djebar, Ces voix qui m'assiègent 111)

La romancière et cinéaste fait un "cinéma de regards " (Djebar, Ces voix qui m'assiègent 161-167). Le regard est toujours quadruple : premièrement, il y a le regard, toujours malveillant, jeté soit par l'ancien colonisateur, soit par les hommes sur l'autre, sur la femme, sur le/la colonisé(e), qui se trouve ainsi dépossédé(e) de son corps et devient un simple « objet »; deuxièmement, il y a le regard de la cinéaste, qui est un regard bienveillant souhaitant redonner à la femme, au colonisé d'hier, son droit de parole ; troisièmement, il y a le regard de la protagoniste qui apprend à regarder ouvertement les autres et se constitue ainsi en sujet, comme Hajila dans le roman Ombre sultane (1987) ; et quatrièmement, Assia Djebar thématise aussi la conversion du regard qu'on doit porter sur soi-même, sur le soi dont il faut se soucier, et cette conversion du regard, c'est ce qu'on appelle écriture.

\footnotetext{
${ }^{2}$ La philosophie est aussi enseignée en latin ; Assia Djebar a même choisi le grec, à défaut de pouvoir choisir l'arabe classique comme deuxième langue étrangère...
} 


\subsection{L'askêsis et l'écriture}

Ce n'est qu'au moment où les femmes ont acquis un certain niveau de maturité, où elles ont acquis la force nécessaire pour regarder l'autre et pour se regarder, qu'elles prennent enfin la plume. Car l'écriture est toujours une entreprise périlleuse, notamment quand on est une femme de sensibilité maghrébine et de langue française, ou quand on est une écrivaine de «l'entre-deux», de "l'entre-des ». Mais la force s'apprend : c'est l'askêsis (comme elle est pratiquée au ${ }^{\text {er }}$ et II ${ }^{\mathrm{e}}$ siècle) qui permet aux femmes d'acquérir quelque chose qui protège le soi et qui permet au sujet de parvenir jusqu'au soi. C'est la paraskeuê (Foucault L'Herméneutique du sujet 306) : la paraskeuê doit leur donner la force d'affronter des situations difficiles et de braver même la mort. L'askêsis est donc tout un ensemble d'exercices qui permettent aux femmes de se doter de cette force.

Ainsi, les femmes apprennent à écouter les autres. C'est ce qu'a fait Assia Djebar dans les montagnes du Mont Chenoua, où elle parle avec les dames honorables de sa tribu maternelle, les Beni Menacer, et les récits des femmes en arabe dialectal et féminin, la relancent sur les voies de l'écriture après un silence d'une dizaine d'années (1967-1980). C'est aussi ce que fait Malika Mokeddem qui, encore enfant, aime écouter sa grand-mère nomade, assise à côté d'elle ou blottie dans son sein, et la vieille dame, la seule à pouvoir dompter la petite fille rebelle, lui fait découvrir de nouveaux horizons. Et Leïla Sebbar apprend aussi à écouter l'autre et à s'écouter : ses conversations avec des Algérien(ne)s, des enfants d'immigré(e)s, des Français(es) qui, que leurs pieds soient noirs ou rouges, se passionnent pour cette terre d'Algérie, avec ses "sœurs étrangères » (Sebbar, Mes Algéries en France. Carnet de voyages 48-59), lui permettent de suturer ce qui a été séparé de manière grave par l'Histoire. Et Nine Moati, au moment d'accoucher de sa première fille, entame, dans son premier roman Mon enfant, ma mère (1974), un étrange dialogue avec sa mère Odette morte trop tôt, pour pouvoir assumer sa propre maternité ${ }^{3}$. Hélé Béji voue une grande admiration à sa grand-mère, lors de ses séjours à Tunis dans la vieille maison où elle a sa chambre, elle s'est volontiers laissée envelopper par le verbe généreux de sa grand-mère, dont elle raconte la vie modeste dans son roman L'œil du jour (1985).

Instinctivement ou tout simplement faute d'argent, les femmes font ce que recommandent les philosophes de l'époque hellénistique et romaine, elles relisent sans cesse les livres pour s'approprier les vérités qu'ils contiennent, comme Malika Mokeddem (Mokeddem, La Transe des insoumis 71-72). Les femmes apprennent aussi à maîtriser leur corps : ainsi, Assia Djebar se met en mouvement, ivre de lumière et de poésie, mais, par une belle journée d'automne, elle manque de mourir sur les rails du

\footnotetext{
${ }^{3}$ Notons que son frère Henry Moati, réalisateur et cinéaste, qui opte pour le prénom de son père Serge, éprouve aussi le vif besoin de retracer dans son roman Villa Jasmin (2003) l'histoire de sa famille et de se rapprocher du père vénéré.
} 
tramway. La jeune fille devient mobile, elle sort, d'abord encore avec le consentement du père qui l'a conduite à l'école, un matin d'automne, qui autorise les sorties de plus en plus irréconciliables avec le rôle traditionnel de la femme arabe au fur et à mesure que la fille grandit. Puis, de plus en plus souvent, à son insu : encore jeune pensionnaire à l'internat à Blida, elle se met à sortir plus tôt que prévu les samedi après-midi pour se promener avec son amie Mag en ville (Djebar, Nulle part dans la maison de mon père 138). Plus tard, elle se met à échanger, pendant sa dernière année à l'internat, des lettres avec Tarik, un jeune étudiant arabisant d'Alger - correspondance pourtant anodine, mais que le père n'aurait jamais autorisée. Celui-ci déchire la première lettre que l'étudiant avait envoyée à l'adresse de ses parents (Djebar, L'Amour, la fantasia 12-13 ; Nulle part dans la maison de mon père 251-252). Jeune étudiante en hypokhâgne, elle se promène, à l'insu du père, avec ce même étudiant, qui deviendra son fiancé, puis son premier mari, à Alger. Un jour, après une terrible dispute avec ce dernier qui voulait entraver sa marche vers la liberté, et ivre de lumière et de mouvement, ivre aussi de poésie arabe et latine (car elle a passé la dernière année à l'internat et sa première année d'études à lire les poèmes arabes que Tarik lui envoyait en y joignant la traduction en français, et la poésie latine au programme), terrifiée à l'idée de devoir avouer un jour ses escapades à son père dont elle craint le verdict, la jeune femme veut se dissoudre dans la mer ou, à défaut, dans l'air de la glorieuse baie d'Alger : dévalant les escaliers menant au port en toute vitesse, elle se couche sur les rails du tramway, et n'est sauvée de justesse de la mort que grâce au conducteur qui réussit à stopper l'engin quelques centimètres devant le corps étendu de la fille. S'enfuir, échapper à une réalité qui lui paraît soudain trop lourde ; et se rendre compte, enfin, qu'on ne peut pas y échapper, qu'il vaut mieux affronter toutes les difficultés, mais comment? En se mettant à écrire, va-t-elle décider trois ans après ce terrible incident en automne 1953, en 1956, quand aura déjà éclaté la guerre d'Algérie. Ainsi, la lecture, l'écriture, les études libèrent définitivement la jeune fille, même si elle doit encore lutter pour enlever successivement tous les voiles métaphoriques qui lui interdisent de dire ouvertement et sans fausse honte « je ».

Malika Mokeddem, pour sa part, devient anorexique. C'est sa façon à elle de libérer son corps :

« Je me gave alors de la seule liberté à ma portée, la lecture. Je lis tout le temps. Avec avidité. Désormais, je peux avoir des livres à profusion. Je suis loin de comprendre tous les mots que je lis et c'est tant mieux. Les mots inconnus sont les plus grandes foulées de ma fugue. Ils me laissent libre du sens accordé à leur graphie, à leur sonorité, et me grisent davantage. Ils représentent tout ce que je ne connais pas de l'histoire, de la géographie, des humains. Ils sculptent mon imaginaire entre le manque et ses tentations. L'habitude du dictionnaire et des atlas posés à proximité ne viendra qu'un peu plus tard. Avec l'exigence de la maîtrise d'une langue et la nécessité de déchiffrer ses résonances en moi. Pour l'heure, j'ai seulement besoin de dévorer l'espace et la substance des mots. Les livres sont maintenant mes seuls vivres. Je suis devenue anorexique. » (Mokeddem, La Transe des insoumis 142-143) 
Leïla Sebbar ramasse sur les trottoirs parisiens des objets disparates témoignant de la présence physique des « autres », des « étranger(e)s », des harkis, des immigré(e)s et de leurs enfants sur le sol français (comme les boîtes de tabac à chiquer, les enveloppes contenant le papier à cigarettes «Le Zouave », les cartes de marabout distribuées aux bouches du métro au 18 arrondissement, les papiers d'oranges froissés...) ; elle prend le métro, toujours à la recherche de tags en arabe, elle voyage en France pour visiter les lieux de la mémoire commune. Ses «vagabondages urbains » lui permettent de se constituer une identité à la fois complexe et complète.

Ces exercices doivent permettre aux femmes de se doter d'une force exceptionnelle : elles doivent être prêtes à affronter des situations difficiles (on y retrouve l'idée de la praemeditatio malorum des stoïciens); elles doivent même braver la mort (on y retrouve la meletê thanatou, ce en quoi la praemeditatio malorum culmine).

\section{L'écriture salvatrice, l'écriture de résistance et la résistance de l'écriture}

Leur formation à l'école française donne envie aux auteures maghrébines de s'inventer une identité complexe tenant compte de leur situation entre plusieurs univers et plusieurs langues. Notons pourtant que, compte tenu que la construction identitaire est une entreprise très difficile aboutissant à un fragile équilibre, ce qu'on appelle l'identification collective l'emporte souvent sur l'individualité. Il s'agit d'une «dépersonnalisation » (Kaufmann 147). Néanmoins, soulignons qu'il est très rare qu'une telle dépersonnalisation soit mal vécue, car, d'habitude, elle s'accompagne de sentiments tendres. On peut sans hésiter parler d'une véritable dépossession de l'individu, lorsque celui, voyant que son conjoint ou son enfant souffre, souffre autant, si ce n'est encore plus que celui-ci : l'individu ne se vit plus comme une entité autonome, il se regarde comme un membre de la famille, plus encore, comme une partie du corps familial : il s'y dissout. Ainsi, Nine Moati a l'impression d'être à jamais attachée au corps de sa mère, malgré sa disparition. Elle en parle dans son tout premier roman Mon enfant, ma mère (1974), où elle entretient un touchant dialogue avec sa mère Odette morte avant l'âge, nommée affectueusement « Man ». Elle constate : «Man, c'est bizarre, j'ai, par moments, l'impression que je suis toi, enceinte de moi... » (82)

Néanmoins, cette dissolution de l'individu dans le corps familial peut aussi être ressentie par celui comme étant en première instance un piège : la sensation d'être soudain pris au piège peut avoir des conséquences catastrophiques pour l'individu, car il est très difficile pour lui d'exprimer son vœu de redevenir plus autonome sans blesser les autres membres de la famille. Aussi doit-il gérer chaque tentative d'émancipation avec beaucoup de délicatesse (cf. Kaufmann 148). Chez Malika Mokeddem, cette dissolution dans le corps familial prend une forme assez concrète : ainsi, durant son enfance, elle a dû partager avec sa nombreuse fratrie la couche commune. En hiver, sa situation s'aggravait encore, car les parents et les enfants 
n'étaient pas seulement alignés côte à côte, mais ils partageaient aussi la couverture, une énorme couverture très lourde de laine rêche tissée à la maison et qui risquait d'étouffer la petite Malika. Elle se réveillait souvent en pleine nuit, parce qu'elle avait la terrible sensation d'étouffer. Encore petite, Malika souffrait d'insomnies, car elle ne supportait pas ce nivellement du corps familial, auquel elle tentait d'échapper en se levant et en allant se réfugier dans les bras de sa grand-mère qui dormait dans la cuisine. Mais pour échapper au corps familial qui risquait de l'engloutir, il lui fallait précautionneusement extirper d'abord ses mains, ensuite sa poitrine, puis démêler ses jambes (Mokeddem, La Transe des insoumis 33).

Ainsi, les identifications collectives (dont la famille représente sans aucun doute le foyer identitaire le plus important) peuvent certes avoir des effets très positifs pour l'individu en donnant sens à sa vie et en lui permettant de restaurer l'estime de soi ; mais, à l'inverse, elles peuvent aussi piéger l'individu, incapable de retrouver son autonomie.

Compte tenu de ces faits, l'écriture devient une véritable explosion dotée d'un effet libérateur, comme un cri. L'écriture des auteures maghrébines est donc aussi, toujours, l'écriture du cri. Ainsi, Malika Mokeddem a, elle-aussi, découvert la force du cri. Elle s'enfermait régulièrement dans la pièce des invités pour échapper à sa mère. Celle-ci, offusquée par ce comportement, voulait la forcer de lui rendre la clef dérobée, mais en vain :

«Je ne restituerai pas de sitôt la clef de la fameuse pièce. Je hurle ou détale quand ma mère
tente de me l'arracher. Mes cris l'inhibent, la tétanisent d'effroi. Sauf à rugir comme une
bête blessée sous les coups de la mort, une fille ne crie jamais, surtout pas de rébellion. Moi,
j'ai appris ça, la force du cri. Je sais son poids de honte et d'interdit. À défaut de me faire en-
tendre, comprendre, je le crois capable de me défendre. J'en ai mesuré l'impact dans les
yeux de ma mère. J'en ai apprécié la puissance répulsive. Il me vrille sur place et c'est ma
mère qui bat en retraite en gémissant : «Elle est folle, ma fille !» " (Mokeddem, La Transe des
insoumis 143)

\section{Malika Mokeddem : le travail ou la liberté qui s'achète}

Dès son entrée au lycée, Malika Mokeddem doit travailler, autre façon de devenir indépendante. D'abord comme pionne à l'internat, pionne et pionnière faisant irruption dans le monde masculin, surveillant les autres filles qui, peu à peu, nostalgiques, cédant aux menaces des hommes qui considèrent les filles qui sortent librement, sans voile, qui font des études, comme de simples dévergondées, cédant au chantage du clan, retournent chez elles pour préparer leur trousseau de mariée et entamer leur vie d'épouse et de mère. Parce que le père et le patriarche sévères et autoritaires ne tolèrent plus les escapades de la jeune fille. Malika Mokeddem est profondément dégoûtée par ces pratiques, et ne peut pas comprendre la soumission de ses camarades si dociles, trop dociles peut-être. Pour sa part, elle ne songerait jamais à quitter l'école 
et à se laisser enfermer, foulard, voile, réduction du corps de la femme à sa fonction procréatrice.

Il s'agit d'un travail ingrat, parce que le directeur de l'établissement scolaire ne manque pas une seule occasion d'humilier et de harceler celle qui ose briser les tabous, et refuse au début de la payer pendant les vacances, renouvelant son contrat à chaque rentrée, la privant ainsi de son maigre salaire pendant les vacances d'été. Mais, signant pour la première fois le contrat de travail, s'installant dans le petit coin qu'on lui avait aménagé dans le dortoir, à l'abri du regard des autres, presque une chambre pour elle seule, elle goûte surtout, malgré les tracasseries, le merveilleux sentiment d'avoir recouvré enfin sa liberté, voire sa liberté initiale : l'adolescente, qui a grandi au bord du désert algérien, sent déjà confusément que toutes les contraintes qui pèsent sur son corps, sont une invention dangereuse des hommes, et que la réalité première des femmes, mais aussi des hommes qui sont tout autant frappés par ces interdits, est cette merveilleuse liberté qu'ont su conserver les nomades et que lui a tant de fois racontée sa grand-mère Zohra. Ce regard qui se détourne de tout ce qui n'est pas le soi :

«[...] il faut aller vers le soi comme on va vers un but. Et là ce n'est plus simplement un mouvement des yeux, c'est un mouvement de l'être tout entier, mouvement de l'être tout entier qui doit se porter vers le soi comme seul objectif. Aller vers le soi, c'est en même temps un retour à soi : comme on revient au port ou encore comme une armée regagne la ville et la forteresse qui la protège. » (Foucault L'Herméneutique du sujet 205)

Ensuite, elle a travaillé comme enseignante de mathématiques pendant ses études de médecine à Oran, travail qui lui permettait de subsister. Finalement, elle a obtenu un poste de médecin à Paris, où la femme algérienne, à une époque où le souvenir de la guerre d'Algérie était encore vivace, souffrait une nouvelle fois d'une discrimination sournoise l'obligeant à quitter l'hôpital et à partir une nouvelle fois : elle s'installe dans le midi de la France et elle retrouve la Méditerranée, qui est un autre désert.

Le travail qu'elle fait lui permet de s'épanouir, certes, si l'on pense à son poste d'enseignante ou à son travail de médecin ; mais, plus encore, il lui permet de devenir financièrement indépendante, chose inouïe à l'époque, où les femmes, les éternelles mineures, dépendaient d'abord de leur père, qui chargeait ensuite son gendre de cette tâche présumée difficile à cause des vices féminins. Mais Malika Mokeddem ne tire pas seulement une joie vaniteuse de la certitude d'avoir secoué le joug de la Tradition, et d'avoir, au terme de tant de luttes éprouvantes, contre son père, contre le clan, contre les «autres", cette instance invisible, mais, semble-t-il, toutepuissante, enfin obtenu le droit de continuer sa libération : visiblement dégoûtée, elle se rappelle dans son autobiographie La Transe des insoumis (2003), le moment où son père, hypocrite, à qui elle a dû remettre tout son salaire de pionne, l'a complimentée : «Ma fille, maintenant tu es un homme ! (165) Comme une esclave, elle doit acheter sa liberté. 


\section{L'amour paternel et l'amour conjugal}

Assia Djebar, Malika Mokeddem, Nine Moati, Leïla Sebbar et Hélé Béji réussissent à s'épanouir pleinement, malgré tous les revers qu'elles doivent subir.

\subsection{Nine, Henry-Serge Moati et le rêve d'un Etat libre et démocratique}

À l'âge de 19 ans seulement, Nine Moati perd ses parents, et doit s'occuper désormais seule de son frère Henry, le dernier d'une fratrie de trois enfants. Ils s'installent à Paris, loin de la Tunisie natale à laquelle Nine continue pourtant de rêver, et qu'elle fait revivre dans ses textes. Le petit Henry, l'enfant choyé, souffre beaucoup de la mort de ses parents : tiraillé par des sentiments contradictoires, il décide que Serge Moati n'est pas mort : il opte pour le prénom de son père et se choisit aussi la carrière de journaliste (Allali). Sa sœur Nine, au moment de sa première grossesse, a aussi la vive impression que sa mère n'est pas morte, mais qu'elle est sa mère, enceinte d'elle... (Moati Mon enfant ma mère 82). Dans son roman Du côté des vivants (2006), Henry Moati raconte son enfance tunisienne, ses obsessions, la mère et le père morts trop tôt, Tunis quitté pour Paris. Sa sœur Nine reste aussi hantée par le souvenir de la Tunisie, du père, de la mère et de leur histoire commune, qu'elle raconte dans son texte autobiographique Mon enfant, ma mère (1974) et aussi dans son roman Deux femmes à Paris (2000). Les orphelins se souviennent, ils maintiennent intacte la chaîne de la transmission, ils racontent la légende du père et de la mère... Ils se souviennent des récits du père et de la mère, racontant le Protectorat, le Destour et le réveil nationaliste en Tunisie, Habib Bourguiba, la répression coloniale violente, l'accession au pouvoir du Front Populaire en 1936 et la libération des chefs indépendantistes, l'enthousiasme du père socialiste, à l'époque à Paris avec sa femme Odette, où il s'est installé après son expulsion, où il travaille comme journaliste au Populaire de Léon Blum, et où le jeune couple vit la période exaltante du Front Populaire. Cependant, ils sont bien conscients que des nuages noirs s'amoncellent à l'horizon, parce que les réfugié(e)s politiques échouant par vagues successives en France, racontent l'Allemagne hitlérienne, la terreur, les persécutions, l'holocauste et les camps de concentration. Les parents se perpétuent dans leurs enfants... Et les enfants (se) racontent l'emprisonnement de Bourguiba en 1938 en France qui dure cinq ans, l'occupation allemande, la terreur et le génocide, les camps de la mort, ces camps où le père était aussi détenu pour ses convictions politiques avant de parvenir à le quitter, ils (se) racontent la Libération, la fierté du père participant à la Libération de Paris avant de retrouver sa famille à Tunis. Ils (se) racontent le 15 mai, quand les Alliés transfèrent l'autorité de la Tunisie à la France libre, qui, sans perdre du temps, arrête des centaines de sympathisants nationalistes et dépose le bey régnant, Moncef Bey, jugé pro-allemand, pour le remplacer par Lamine Bey, provoquant la colère des Tunisien(ne)s qui ont majoritairement soutenu la reconquête alliée ; ils (se) racontent le déclin de la dynastie beylicale, 1945, quand le Général de Gaulle propose à la Tunisie 
le statut «d'Etat associé » au sein de l'Union française, l'entêtement de Bourguiba qui, de retour au pays en 1949, au lieu d'accepter l'offre française, intensifie sa campagne pour l'indépendance de la Tunisie, en s'appuyant notamment sur l'Union générale des travailleurs tunisiens (UGTT), l'arrestation de Bourguiba et des chefs nationaliste et la dissolution forcée du gouvernement Chenik en 1952, la lutte armée et la «Main rouge », cette organisation terroriste créée par des colons extrémistes qui ne veulent pas abandonner la Tunisie aux Tunisien(ne)s parce qu'ils ne veulent pas perdre leurs privilèges, car, comme l'a constaté fort correctement Albert Memmi, tout colonisateur est privilégié (Memmi 35-38); ils (se) racontent les attentats et les émeutes, l'engrenage de la violence menant la Tunisie au bord d'une guerre ; ils (se) racontent le 31 juillet 1952 et la visite de Pierre Mendès France, le nouveau président du Conseil français, en Tunisie, promettant l'autonomie interne, la fin des émeutes, le 3 juin 1955 et la signature d'une série de conventions consacrant l'autonomie tunisienne, par le premier ministre tunisien Tahar ben Amar et le successeur de Mendès France, Edgar Faure. Ils (se) racontent le 17 septembre et le premier gouvernement composé exclusivement de Tunisiens depuis 74 ans (1881-1955), le 20 mars 1956 et la souveraineté de la Tunisie, les premières élections législatives de l'histoire tunisienne organisées le 25 mars et la victoire du Néo-Destour, Bourguiba élu président de la première Assemblée nationale tunisienne, Bourguiba nommé Premier ministre, la déposition du bey le 25 juillet 1957 et la proclamation de la République. Nine et Henry-Serge perpétuent les convictions politiques du père socialiste et laïc, qui se considérait comme un Tunisien juif plutôt que comme un Juif tunisien, parce qu'il aimait son pays et voulait participer à l'édification d'un Etat libre, démocratique et laïc, même s'il pressentait déjà que ses espoirs seraient déçus :

\begin{abstract}
« Pas un mot... Ils n'ont pas dit un mot pour nous remercier! Pas un mot de remerciement aux militants qui ont soutenu la cause tunisienne ! Rien! Nada! Cheï ! Rien que des cocoricos nationalistes! Nul! Pour ces messieurs, tous en chechia, rien ne compte plus que la Tunisie au chaud dans ses frontières avec son " parti unique », et ses «masses » qui obéissent au doigt et à l'œil ! Un petit Etat de quatre sous, replié sur lui-même ! Frontières, miradors, passeports, visas, jeunesse au pas cadencé, femmes aux youyous synchronisés, slogans et applaudissements à la commande ! Ils vont faire un pays avec l'islam comme religion officielle, ils nommeront Habib « Président à vie » et le tour est joué ! Ah, crois-moi, Odette, moi je rêvais d'autre chose que de cette Indépendance-là, où les nouveaux petits chefs vont remplacer les anciens, mot à mot, trait pour trait. Non, j'oubliais! Seules les djellabas remplaceront, dans les pince-fesses, les costumes trois-pièces, et le ramadan deviendra obligatoire! Tristesse ! À crever ! Et la liberté ? Et le socialisme ? Et la démocratie? Au trou toutes ces fariboles! Au trou ! C'était juste bon pour les naïfs de mon espèce. Un jour, tu verras, Odette, on sera des étrangers ici ! (Moati Serge, Du côté des vivants 299)
\end{abstract}

\title{
5.2 Hélé Béji et l'entreprise dangereuse de l'écriture
}

Agrégée de lettres modernes, Hélé Béji a d'abord enseigné la littérature à l'Université de Tunis. Ensuite, elle a occupé un poste de fonctionnaire international à l'UNESCO. Elle a donc fait son chemin dans un monde pas toujours favorable aux femmes. No- 
tons pourtant que ce n'est pas tant son travail d'universitaire et de fonctionnaire qui la met en danger, mais son écriture : tout en étant présente dans la sphère publique, tout en prenant ouvertement la parole lors des conférences et sommets, elle ne parle jamais d'elle-même, elle parle pour les autres, et sa présence dans la sphère publique est donc justifiée :

\footnotetext{
"Cette présence [de la femme dans la sphère publique, R.G.] reste anormale et doit être justifiée. Tous les discours le disent, ceux de l'Etat, ceux des familles : la femme sort pour travailler, $c^{\prime}$ est utile à la construction du pays, elle sort pour militer (elle a participé à la guerre [en $\mathrm{Al}$ gérie notamment; en Tunisie et au Maroc, la femme a participé aux émeutes et soutenu ainsi le mouvement indépendantiste, R.G.l). Elle ne sort donc pas pour son plaisir et doit faire attention de rester dans la sphère publique une femme, garder sa pudeur, sa vertu, toujours soupçonnée, rester au service de la communauté et passer inaperçue. » (Gadant 95)
}

Mais dès qu'elle prend la plume pour écrire de la littérature, elle s'expose au regard des autres, et aussi de ceux et celles qui condamnent sévèrement ce dévoilement, cette «mise à nu». On voit donc à quel point l'écriture littéraire est une entreprise dangereuse... Ecrire, c'est donc se révolter. Se révolter contre le sort cruel que la tradition réserve aux femmes. Se constituer un corps androgyne. Dans son essai Une force qui demeure (2006), où se mêlent des passages autobiographiques et des analyses pertinentes de la situation de la femme maghrébine, elle constate : "Je savais que je n'étais pas un garçon, mais j'étais persuadée aussi de ne pas être de leur sexe à elles [...]. » (Béji, Une force qui demeure) Mais est-ce vraiment une révolte ? Ne s'agit-il pas plutôt d'une légitime révolution?

\subsection{Assia Djebar et le français comme «langue du père, "langue androgyne » et « langue du sang"}

C'est son père qui a conduit Assia Djebar à l'école, et qui lui a donc permis de conquérir, à petits pas, l'espace masculin. Mais en même temps, elle a grandi dans un harem, c'est-à-dire au milieu des femmes voilées qui ne sortaient que pour aller au hammam. Comme Hélé Béji, Assia Djebar a donc la vive impression qu'elle est un « être androgyne » : « [...] Dans ce livre [L'Amour, la fantasia, R.G.], j'étais dans le rapport entre le français et l'arabe, le français m'ayant apporté la liberté du corps, à onze ans. Mais c'était aussi une tunique de Nessus. [...] à seize ans, dix-sept ans, je me concevais dehors autant en garçon qu'en fille. » (Gauvin 30)

D'une part, Assia Djebar considère le français comme la « langue du père » et comme la langue de l'école, son premier espace de liberté. Mais d'autre part, il ne faut pas oublier que le français est aussi la langue de la violence, des envahisseurs et de l'ancien colonisateur, qui a "désarabisé » les Algérien(ne)s en confisquant les biens habous ou waqf prenant en charge beaucoup de lieux de l'enseignement traditionnel du Coran et de la langue arabe, mais qui, ensuite, n'a pas fait de sérieux efforts pour les «franciser », le terme de francisation ne désignant que des mesures visant à rem- 
placer les toponymes arabes par des toponymes français ou à les franciser phonétiquement ou à franciser les patronymes. Au regard de ces faits, il n'est pas étonnant que le français soit aussi la « langue du sang » pour Assia Djebar, comme elle le constate elle-même dans son livre Ces voix qui m'assiègent: "Par ailleurs, pour moi, ma langue d'écriture me fut tout récemment langue du père («fillette de six ans allant à l'école, main dans la main du père »). Or celle-ci m’est aussi pour moi, Algérienne, langue des envahisseurs et des soldats, langue du combat et des corps à corps virils, en somme langue du sang. » (148-149) Tout en écrivant en français, elle n'oublie jamais que l'implantation de la langue française en Algérie « s'appuie sur la mort » des siens. De ce dilemme résulte le problème de la romancière de dire l'amour en français. C'est un problème qu'elle essaie de résoudre en 1985 en publiant son roman L'Amour, la fantasia. Dans ce roman, elle recourt à l'homophonie suivante pour rendre compte de ses difficultés à écrire des mots d'amour en français : «L'amour, ses cris » (« s'écrit »). (299)

\subsection{Leïla Sebbar et les blancs de l'Histoire}

Leïla Sebbar, qui n'a pas appris la langue de son père, l'arabe parlé, reste pourtant hantée par cette langue, qui devient une véritable «langue fantôme » qui s'inscrit en creux dans ses textes, comme un " chant secret » (Sebbar, L'arabe comme un chant secret). L'arabe est bien la «langue du sang », la langue de la brutalité, la langue des garçons arabes qui, infatigables, insultaient les trois « petites Françaises » sur le chemin d'école, mais c'est aussi la langue du père, de la famille algérienne et la langue de la beauté.

Leïla Sebbar est certes en exil où qu'elle aille, parce que l'exil s'hérite, et elle a hérité de l'exil du père et de la mère. L'exil est le lieu de tous les manques. Mais elle a compris que l'exil est aussi le lieu de tous les possibles. Et qu'elle a besoin des manques, des non-dits, des blancs pour écrire : «Il faut que je fasse attention, parce que... parfois j'ai peur que ce travail de... d'aller comme ça au plus près du réel... arrête l'inspiration... empêche la fiction. Parfois, ça me fait peur. », nous a-t-elle confié lors de notre deuxième rencontre à Paris en septembre 2007.

\subsection{Malika Mokeddem : l'amour maternel et l'amour paternel - un amour impos- sible?}

Malika Mokeddem n'a pas de père instruit, voire même de père instituteur de français comme Assia Djebar et Leïla Sebbar, qui n'aurait jamais songé à interrompre arbitrairement la scolarité de sa fille. Elle doit lutter pour échapper à la claustration, d'abord quand elle a la chance de poursuivre ses études dans la ville voisine, mais quand il faut d'abord convaincre le père (elle y réussit grâce à sa maîtresse d'école), et ensuite quand le patriarche du clan décide de la marier, et le père n'ose pas s'y opposer. Le père de Malika Mokeddem n'arrive pas à briser les tabous et à être le mari 
et le père aimant qu'il aurait pu être. Il n'hésite même pas à mentir à sa fille et préfère ouvertement ses descendants mâles à ses filles, croyant sans doute respecter ainsi la «tradition ». La mère, analphabète, cloîtrée dans un gourbi, puis, quand le mari obtient enfin un travail mieux payé comme gardien d'un puits, dans la maison au pied de la dune, croit avoir trouvé un remède à son éternelle oppression, en accouchant à intervalles réguliers d'un enfant: parce qu'elle est la mère de ses enfants, la mère de ses fils surtout, son époux si sec et autoritaire doit enfin l'écouter, même s'il n'est toujours pas obligé de tenir compte de ses conseils. La mère se révèle incapable d'aimer tendrement sa fille Malika, la rebelle qui ne se pliera jamais au le joug de la tradition et n'acceptera jamais le rôle de l'épouse obéissante et de la mère comblée qu'elle est pourtant censée assumer. Malika ne sait pas comment aimer son père et sa mère, cette mère qui garde un secret si terrible qu'il lui faudra tant d'années pour le raconter (Mokeddem, Je dois tout à ton oubli 2008). Mais quand elle les perd, elle souffre : peut-être parce qu'elle n'a jamais eu la chance de les aimer?

Sur le plan affectif, sa relation amoureuse avec Jean-Louis, un Français dont, audace suprême, elle accepte la demande en mariage, la fait parvenir peu à peu à sa maturité : elle se sent merveilleusement protégée. Pendant les dix-sept ans que Jean-Louis partagera sa vie, le couple réussira à créer un nid douillet à Marseille, ils feront construire cette maison sublime au bord d'une falaise, et c'est Malika Mokeddem qui décidera de la disposition des terrasses, de l'aménagement du vaste jardin, créant ainsi un véritable havre de paix où la femme, aussi assoiffée que jadis, peut recouvrer ses forces après une journée de travail harassant. Elle passe avec son mari, amateur de la voile, les étés sur leur bateau, baptisé «Vent de sable». Malika Mokeddem éprouve le vif besoin de se retirer des journées entières du monde des vivants pour écrire, pour raconter les mort(e)s et les vivant(e)s, les jours passés et les terreurs présentes. Elle éprouve un besoin excessif de liberté et de solitude, même si elle comprend l'angoisse de Jean-Louis qui veut la retenir. Mais pour aller vers elle-même, il lui faut quitter son amour.

\section{6. « Tricher la langue»}

Pour la femme maghrébine, il est donc très difficile de prendre la plume, car l'écriture est chargée d'un énorme poids d'interdits et de tabous. La tentative de la femme de s'en emparer, signifie qu'elle porte atteinte au code d'honneur et qu'elle vise même à bouleverser la société voire même à détruire ce qu'on considère comme un «équilibre » (aux hommes le dehors, aux femmes la maison), mais qui se trouve être, en réalité, un déséquilibre.

Barthes propose de «tricher avec la langue » et de «tricher la langue », pour « entendre la langue hors-pouvoir»: "Cette tricherie salutaire, cette esquive, ce leurre magnifique, qui permet d'entendre la langue hors-pouvoir, dans la splendeur d'une révolution permanente du langage, je l'appelle pour ma part : littérature. »(15) Ré- 
gine Robin se rend compte que les écrivain(e)s qui sont «fixé[e]s à une langue » (comme les écrivaines de sensibilité maghrébine qui n'écrivent qu'en français, qui est devenu pour elles un merveilleux terrain neutre leur permettant de dire ce qu'elles ne pourraient pas dire en arabe, la langue du Coran et de la tradition arabomusulmane, ou comme les écrivaines de «l'entre-des»comme Leïla Sebbar, qui n'ont pas appris l'autre langue), éprouvent pourtant le vif besoin de faire entendre dans leurs textes toutes les langues qui, présentes en dépit de leur absence, sont des composantes centrales de leur identité et dont les sonorités les hantent: les écrivain(e)s «[...] ne pouvant sortir [d'une langue], s'en [font] une contre-langue [...]» (Robin 18); ils/elles «[font] migrer les langues dans [cette langue] » (18), ils/elles « [...] brise[nt la langue, ici le français, R.G.] pour s'y inscrire en creux. [Ils/elles] désarticule[nt] la syntaxe, injecte[nt] dans [leur texte] des mots étrangers [...], utilise[nt] des citations, une intertextualité poétique qu'[ils/elles] remodèlent. » (18-19)

C'est la poésie qui leur permet d'entendre et de faire entendre la «langue horspouvoir» (Barthes 15). La poésie leur donne la possibilité d'inscrire les rythmes de l'autre langue, les rythmes du corps (Assia Djebar) et le rythme primitif (1-2) de la marche des nomades (Malika Mokeddem) dans leur texte. Témoin notamment le poème «Sistre » d'Assia Djebar, qui, avec ce poème où elle imite le mouvement rythmique des corps des amants, réussit à surmonter son aphasie amoureuse : «[...] le corps recherche sa voix, comme une plie remontant l'estuaire. [...] puis le chant long, le chant lent de la voix femelle luxuriante enveloppe l'accouplement. » (Djebar, L'Amour, la fantasia 156-157)

«Long silence, nuits chevauchées, spirales dans la gorge. Râales, r ruisseaux de sons précipices, sources d'échos entrecroisés, cataractes de murmures, chuchotements en taillis tressés, surgeons susurrant sous la langue, chuintements, et souque la voix courbe qui, dans la soute de sa mémoire, retrouve souffles souillés de soûlerie ancienne. » (Djebar, L'Amour, la fantasia 156)

Dans ce poème, Assia Djebar utilise l'allitération et l'assonance pour inscrire l'autre langue (l'arabe féminin) dans son texte : ainsi, on constate une abondance des consonnes [s] ("Sistre», silence, spirales, ruisseaux, sons précipices, sources, tressés, surgeons, susurrant, sous, souque, soute, souffles souillés de soûlerie) et [z] (entrecroisés), [R] (spirales, râles, ruisseaux, précipices, sources, entrecroisés, cataractes, tressés, surgeons susurrant, courbe, mémoire, retrouve, soûlerie), [J] (chevauchés, chuchotements, chuintements) et [3] (gorge, surgeons). Et on constate une abondance des voyelles [o] (chevauchées, échos, chuchotements), [0 ] (gorge), et [õ] (long, sons, surgeons), [a] (spirales, cataractes), $[\alpha]$ (râles) et [ã] (silence, chuchotements, susurrant, langue, ancienne). Vers la fin du poème, on note aussi une accélération du rythme, jusqu'à ce soit enfin atteint le point culminant du plaisir sexuel :

« De nouveau râles, escaliers d'eau jusqu'au larynx, éclaboussures, aspersion lustrale, sourd la plainte puis le chant long, le chant lent de la voix femelle luxuriante enveloppe 
l'accouplement, en suit le rythme et les figures, s'exhale en oxygène, dans la chambre et le noir, torsade tumescente de «forte » restés suspendus. » (Djebar, L'Amour, la fantasia 156157)

Dans son roman Les Hommes qui marchent (1990), l'écrivaine algérienne Malika Mokeddem retrouve le rythme primitif (1-2) de la marche des nomades :

\begin{abstract}
«Un pas, deux pas. Avec allégresse, le pied retrouvait la plasticité du sable. Délivré de ce sinistre environnement, le corps recouvrait sa souplesse. Un pas, deux pas. Sans voile, un magroune vaporeux flottant sur sa robe à grands volants et les mains nouées dans le dos, Zohra marchait en rêvant. Un pas, deux pas. Tête haute, sens aux aguets, elle observait à la dérobée la vie des citadins. Quand aucun but précis ne les animait, les hommes se regroupaient dehors et attendaient, mollement, que passât le temps. Temps du silence. Silence des fantasmes, de l'inavouable. Un pas, deux pas. » (71)
\end{abstract}

Les auteures maghrébines de langue française sont très sensibles au «tourment de langage » (Glissant 111, 112), parce qu'ils/elles grandissent dans une région géographique où il y a une langue dominante (le français pendant l'époque de la colonisation, aujourd'hui l'arabe de l'arabisation qui doit remplacer le français dans tous ses usages, au détriment des langues parlées), et des langues dominées (l'arabe parlé, féminin, et le tamazight). Ancrées non seulement dans la tradition maghrébine, tradition en grande partie orale, mais aussi occidentale, véhiculée par l'écriture et l'école, elles doivent prendre en compte ces deux composantes contradictoires de leur socialisation, et ensuite les mettre en réseau. Ainsi, elles introduisent des séquences de discours plus ou moins longues (pouvant aller d'un seul mot à plusieurs phrases) de leur langue maternelle dans un texte qui, en apparence, n'est écrit qu'en français (cf. les phénomènes de l'alternance de code - code switching et du mélange de code code mixing ${ }^{4}$; elles inscrivent la sonorité de leur(s) langue(s) maternelle(s), de l'arabe parlé ou du tamazight, dans la chair de leur langue d'écriture, le français (cf. le jeu des allitérations, des assonances, le jeu avec les métaphores de l'arabe); elles «brisent » (Robin 18-19) leur langue d'écriture, la morcellent afin de pouvoir y inscrire les souffrances de leurs consœurs et confrères, et les leurs. Citons l'exemple suivant tiré du roman Le Siècle des sauterelles (1992) de l'écrivaine algérienne Malika Mokeddem. Il s'agit d'un long monologue de Mahmoud qui y apostrophe la mort pour la tenir à distance. On peut constater un jeu subtil avec des métaphores de l'arabe qui sont transposées en français. On y trouve aussi, vers la fin, l'allégorie de la mort et l'allégorie de la poésie. Puis, on peut y constater plusieurs effets de rime (grâce à la paronomase) :

\footnotetext{
${ }^{4}$ Pour plus d'informations, vous êtes prié(e)s de vous reporter à :

Boukous, Ahmed. « Bilinguisme, diglossie et domination symbolique ». Du Bilinguisme. Denoël, 1985. 39-54.

Fishman, Joshua A. Sociolinguistics: a brief introduction. Rowley, Mass.: Newbury House Publ., 1971. - Sociolinguistique. Paris : Nathan, 1971.

Taleb-Ibrahimi, Khaoula. Les Algériens et leur(s) langue(s). Eléments pour une approche sociolinguistique de la société algérienne. Alger : Editions El Hikma, 1997.
}

(C) Copyright Alternative Francophone \& the author 2009 
«Je veux retrouver les steppes et l'humilité, la sobriété de leur horizontale nudité, leur langue de silence. Je ne veux plus chevaucher comme une imagination débridée. Je veux marcher. Marcher comme écrire. Écrire les pas des mots, les mots des pas, sur ces seuils hauts, les plateaux, socle du désert. Et dans la quiétude de l'écrit, sur ces lieux ouverts, je ne veux entrer dans rien mais tout embrasser, à la fois. Je veux débarrasser ma vie de ses fardeaux. Je la veux comme un seuil, ma vie, ouverte et traversée de contrastes. Je la veux tissée d'écrits, métissée dans sa mémoire par toutes ses admirations et par l'oralité. Je la veux mosaïque, scintillante de différences. À l'aune généreuse de la poésie, elle va déplier ses atours, déployer ses attraits et s'offrir chaque instant comme conquête recommencée. Mais je ne la laisserais pas me duper non plus. Les mots et leur dérision, la marche et son exigence sur les hauts plateaux et le désert la maîtriseront, me protégeront de ses excès aussi. Et d'ailleurs, dans ces "nulle-part " avec leur lumière d'éternité, la mort est la mesure du temps et le grain de sable, son unité. De grain de sable en grain de sable, une mise en garde, qui se perpétue à l'infini et effrite toute amorce de vanité. La mort n'est que l'ultime borne avant le franchissement d'un autre seuil. La mort n'est que le plus bref des cauchemars qui me reste à vivre. Mais alors que souvent la seule approche du sommeil me griffe déjà de son glacial frisson, je veux pouvoir me moquer d'elle, la mort. Je veux pouvoir répudier sa peur, parfois l'appeler et la peler de ses voiles d'épouvante. Sans le mystère de sa tragédie, dame solitaire, écrasée par sa propre fatalité, je veux pouvoir parfois me perdre pour elle en effusions. Et, infidèle, je veux m'endormir dans ses bras sur la couche de ma plus belle muse, Poésie. » (Mokeddem, Le Siècle des sauterelles 113-114)

En ce qui concerne la mise en réseau des langues, l'art de la traduction joue un rôle de première importance. La traduction permet une interpénétration subtile des différentes poétiques et sensibilités des langues. Ainsi, Malika Mokeddem a certes transcrit fidèlement les paroles en arabe parlé de sa grand-mère Zohra, mais elle doit les traduire et ce faisant rendre compte de la poésie nomade, arabe, orale de sa grandmère dans la langue de Molière et par l'écrit. Paradoxalement, c'est justement leur transcription, leur «fixation à l'écrit » qui permet aux mots de sa grand-mère nomade de se mettre enfin en mouvement, les mots écrits étant mobiles parce qu'ils peuvent franchir tous les obstacles. Dans son roman L'œil du jour (1985), Hélé Béji retrouve le verbe généreux, splendide de sa grand-mère chérie, illettrée, qu'elle transcrit tout en le traduisant. Assia Djebar transcrit aussi fidèlement les paroles de ses consœurs arabes, tout en les traduisant, ou en les voilant de nouveau : "Petite sœur étrange qu'en langue étrangère j'inscris désormais, ou que je voile. » (Djebar, L'Amour, la fantasia 201) Les écrivaines utilisent plusieurs figures de style pour rendre compte des caractéristiques de l'arabe féminin, comme la litote, la périphrase, l'apostrophe, l'homophonie/la paronomase (cf. l'extrait précédent) et l'allégorie (cf. Mokeddem Le Siècle des sauterelles et La Nuit de la lézarde, qui sont un vibrant hommage au roman Nedjma de Kateb Yacine), et elles décrivent les gestes qui accompagnent les paroles :

- Litote : La litote (soulignée dans l'extrait ci-dessous) témoigne de la grande discrétion des femmes :

" On parle des gens qu'on aime bien, "c'est le miel de la terre, cet homme-là », ou au contraire de ceux qu'on veut éloigner de soi, la pire des terreurs étant suscitée par l'Envieux,

(C) Copyright Alternative Francophone \& the author 2009 
dont on se prémunit par des vade retro et des grimaces pour exorciser l'infamie, une torsion particulièrement sinistre de la bouche, appuyée d'une saillie du globe oculaire où l'on simule l'étincelle crapuleuse, pendant que la main pirouette plusieurs fois pour signifier quelque chose comme " la noirceur de son cœur a déserté son visage », ou encore « il n'a pas une bonne tête de musulman » [nous soulignons] » (Béji, L'œil du jour 115)

- Périphrase : Leur pudeur interdit aux femmes de dire ouvertement l'amour, et même « l'amour imposé », le viol :

"- Ma fille, y a-t-il eu « dommage»?

L'une ou l'autre des aïeules posera la question, pour se saisir du silence et construire un barrage au malheur. La jeune femme, cheveux recoiffés, ses yeux dans les yeux sans éclat de la vieille, éparpille du sable brûlant sur toute parole: le viol, non dit, ne sera pas violé. Avalé. Jusqu'à la prochaine alerte. » (Djebar, L'Amour, la fantasia 283)

\section{- Apostrophe :}

« Hadda,

note suraiguë qui devint râle transpercé...

« Le sanglot, est-ce déjà le sanglot ?... se dit plaintivement Aïcha.

Vaincue par l'émoi esthétique, elle se mit enfin à pleurer.

- Hadda - reprit la chanteuse, un peu plus bas - ouvre-nous la voie royale de délivrance !... Hadda muette, parle-nous!

Et le cri fusa. Spasmodique. Long mais sans puissance. Comme un gargouillis que la mélopée de l'assistance, plus vigoureuse, noya tout à fait : invocations traditionnelles, nom du Prophète, désordre des chœurs... » (Djebar, Femmes d'Alger dans leur appartement 111-112)

\section{- Le langage du corps :}

"Ses descriptions méticuleuses et baroques qui enrichissent l'événement le plus insignifiant de la journée [...], peuvent prendre en quelques instants, dans sa bouche burlesque où son instinct des choses et son don verbal sortent d'un même œuf, les proportions d'un drame aussitôt révélé que résolu, le rythme d'une chronique où se glissent par intervalles les poussées exclamatives, les grands et majestueux soupirs articulés du cœur. Un tapotement bruyant de la main sur la poitrine relaye les mots par une gestuelle plus violente quand ceux-ci, après avoir tout exprimé tout raconté, entrent dans le silence de la stupéfaction, animée par la main qui frappe alors contre soi avec l'intensité d'un discours, arrache encore un rire à l'incroyable, avec les mouvements d'un chef d'orchestre aux dernières notes d'un opéra. » (Béji, L'œil du jour 48-49)

\section{Conclusion : la littérature de «l'entre-deux » ou de « l'entre-des »}

Bien avant les autres, les auteures plurilingues ont compris (sans doute parce que les voix, les langues, toujours au pluriel, sont leur outil de travail et la matière qu'elles travaillent), que nous tous et toutes allons et venons entre les langues, entre les pôles qui existent dans le tissu linguistique des humains : sans cet « entre-les-langues » selon l'expression d'Assia Djebar (Djebar, Ces voix qui m'assiègent 30) ou, dans les termes du sociolinguiste Philippe Blanchet, cet « entre-les-pôles », sans cette " entredeux, cette bordure » (Robin 11), sans cette «frontière », qui est le lieu de tous les possibles (Sebbar, Geyss «L'utopie de l'écriture. Entretien avec Leïla Sebbar. Paris, le 27 septembre $2007 »)$, où nous nous positionnons tous et toutes, l'apprentissage 
d'une autre langue ne serait pas possible, ni l'écriture, d'ailleurs, du moins pas comme les auteures sensibles à l'altérité veulent la pratiquer, et qui est un jeu subtil avec toutes les langues. L'écriture est une utopie, un nulle part, car...

«[...] l'écriture désinstalle, dématernise, déterritorialise, arrache à l'enracinement, creuse un écart, rend visible la perte, la castration symbolique, le manque. L'écriture serait trajet, parcours, cette objectivation qui viendrait à tout instant rappeler qu'il y a de la perte, qu'on n'écrit jamais que dans cette perte, que rien ne viendra combler le manque, mais que l'acte d'écrire, l'impossibilité d'écrire dans l'écriture même est la tentative toujours déçue et toujours recommencée de déjouer la perte, l'apprivoiser, la mettre à distance ; la tentative de saturer, de suturer tout en sachant que l'on ne peut y arriver.

Ecrire c'est toujours jouer, déjouer la mort, la filiation, le roman familial, l'Histoire. » (Robin 11)

À défaut d'être une entreprise solitaire, l'écriture, cette «tricherie salutaire de la langue », est toujours une entreprise solidaire. Au lieu de parler d'écrivains ou d'écrivaines francophones et donc de tracer des frontières artificielles entre des auteurs ou des auteures venant de pays et de cultures différents et écrivant dans des langues différentes, il faudrait plutôt parler d'écrivains ou d'écrivaines plurilingues, de l'entre-deux, de l'entre-des, sensibles à l'altérité. 


\section{Bibliographie}

- Allali, Jean-Pierre. « Lectures. Du côté des vivants. Par Serge Moati ». 10/06/2009. $<$ http://www.crif.org/?page=sheader/detail\&aid=8071\&artyd=8>

- Barthes, Roland. Leçon. (Leçon inaugurale de la chaire de sémiologie littéraire au Collège de France, prononcée le 7 janvier 1977). Paris : Seuil. Points essais, 1989.

- Barthes, Roland. "Inaugural lecture." A Barthes Reader. Ed. Susan Sontag. New York: Hill and Wang, 1982 [1978].

- Beauvoir, Simone de. Le deuxième sexe. Tome I : Les faits et les mythes. Tome II : L'expérience vécue. Paris : Gallimard, 1949.

- Béji, Hélé. L'œil du jour. Maurice Nadeau, 1985.

- Bellon, Guillaume. «Discours, savoir et pouvoir : Roland Barthes et Michel Foucault au Collège de France ». Conférence à l'Institut français de Vienne, le 8 juin 2009. - Bouchentouf-Siagh, Zohra. « «Ici - là-bas » : la trame de soi dans La Transe des insoumis (2003) de Malika Mokeddem ». Bererhi, Afifa (coord.). L'autobiographie en situation d'interculturalité (tome I et II). Blida : Éditions du Tell, 2004, tome II. 331-338. Boukous, Ahmed. «Bilinguisme, diglossie et domination symbolique ». Du Bilinguisme. Denoël, 1985. 39-54.

- Calle-Gruber, Mireille. « Refaire les contes dans la langue adverse. Assia Djebar, Oran, langue morte ». Ruhe, Ernstpeter. Assia Djebar. Studien zur Literatur und Geschichte des Maghreb. Band 5. Würzburg: Königshausen \& Neumann, 2001. 157-167. - Chaulet-Achour, Christiane. «Malika Mokeddem : Écriture et implication ». Algérie Littérature / Action, no 14, octobre 1997. 185-199.

- Chikhi, Beïda. Les romans d'Assia Djebar. Alger : Offices des publications universitaires, 1990.

- Clerc, Jeanne-Marie. Assia Djebar : écrire, transgresser, résister. Paris/Montréal :

L'Harmattan, 1997.

- Djebar, Assia. Nulle part dans la maison de mon père. Paris : Fayard, 2007.

- Djebar, Assia. La Femme sans sépulture. Paris : Albin Michel, 2002.

- Djebar, Assia. « Idiome de l'exil et langue de l'irréductibilité ». 2000.

$<$ http://www.remue.net/cont/ Djebar01.html>

- Djebar, Assia. L'Amour, la fantasia. Paris : Lattès, 1985 ; Albin Michel, 1995.

- Djebar, Assia. Femmes d'Alger dans leur appartement. Paris : des femmes, 1980.

- Djebar, Assia. La Nouba des femmes du Mont Chenoua (film, long métrage), 1978.

- Djebar, Assia. Les Alouettes naïves. Première publication : Paris : Julliard, 1967 ; Actes Sud : Babel, 1997.

Fishman, Joshua. A. Sociolinguistics: a brief introduction. Rowley, Mass.: Newbury House Publ., 1971.

- Fishman, Joshua. Sociolinguistique. Paris : Nathan, 1971.

- Foucault, Michel. L'Herméneutique du sujet. Cours au Collège de France, 1981-1982.

Paris : Gallimard/Seuil, Hautes Études, 2001. 
- Foucault, Michel. Il faut défendre la société. Cours au Collège de France, 1976. Paris : Gallimard/Seuil, Hautes Etudes, 1997.

- Foucault, Michel. Society must be defended. Lectures at the College de France, 19751976. Trans. David Macey. Ed. Mauro Bertani and Alessandro Fontana. Allen Lade, imprint of Penguin Books.

- Gauvin, Lise. «Les langues du roman : du plurilinguisme comme stratégie textuelle ». Gauvin, Lise (sous la supervision de). Les Langues du roman. Du plurilinguisme comme stratégie textuelle. Les Presses de l’Université de Montréal, 1999. 7-14. - Gauvin, Lise. «Introduction. D'une langue l'autre. La surconscience linguistique de l'écrivain francophone ». Gauvin, Lise : L'Écrivain francophone à la croisée des langues. Entretiens. Paris : Editions Karthala, 1997. 5-15.

- Gauvin, Lise. «Territoires des langues ». Entretien avec Assia Djebar. Gauvin, Lise : L'Écrivain francophone à la croisée des langues. Entretiens. Paris : Editions Karthala, 1997. 17-34

- Geyss, Roswitha. « Réflexions sur le rapport entre langue(s) et identité(s) dans la littérature maghrébine féminine de langue française : 'Babel en spirale' et comment les auteures arrivent à 'apprivoiser Babel' ». Philippe Blanchet - Attika-Yasmine Kara - Malika Kebbas - Claude Cortier - Jacqueline Billiez - Khaoula Taleb-Ibrahimi - Safia Asselah-Rahal - Thierry Bulot (comité de publication) : Actes du colloque "Influences et enjeux des contextes plurilingues sur les textes et les discours ", organisé par l'ENS LSH Alger-Bouzaréah et le CREDILIF de l'Université Rennes 2 à l'ENS LSH AlgerBouzaréah du 18 au 20 novembre 2008. À paraître prochainement.

- Geyss, Roswitha. «Briser le silence de la langue paternelle : le projet autobiographique de Leïla Sebbar ». Condei, Cecilia et Cristiana Teodorescu (éds.) : Métissages linguistiques et littéraires. Craiova : Editura Universitaria, 2009

- Geyss, Roswitha. « Réflexions sur le rapport entre langue(s) et identité(s) dans la littérature maghrébine féminine de langue française : le cas d'Assia Djebar et de Leïla Sebbar ». Analele Universitatii din Craiova. Seria Stiinte Filologice. Linguistica. Craiova: Editura Universitaria, décembre 2008. 189-229.

- Geyss, Roswitha. «L'utopie de l'écriture. Entretien avec Leïla Sebbar. Paris, le 27 septembre 2007 ». À paraître prochainement.

- Geyss, Roswitha. Bilinguisme littéraire et double identité dans la littérature maghrébine de langue française : le cas d'Assia Djebar et de Leila Sebbar. Vienne : Mémoire de Magister, 2006. Limag Charles Bonn. 19/12/2008<www.limag.com/Theses/Geyss/Geyss Bilinguisme.pdf>

- Geyss, Roswitha. «L'arabe en accent. Entretien avec Leïla Sebbar. Paris, le 16 mai 2005 ». Geyss, Bilinguisme littéraire et double identité dans la littérature maghrébine de langue française 377-400.

- Glissant, Edouard. Introduction à une poétique du divers. Paris : Gallimard, 1996.

- Grandguillaume, Gilbert. Arabisation et politique linguistique au Maghreb. Paris : Maisonneuve \& Larose, 1983. 
- Grandguillaume, Gilbert, « Le multilinguisme dans le cadre national au Maghreb ». In : Laroussi, Fouad : Plurilinguisme et identités au Maghreb. Publications de 1’Université de Rouen no 233, 1997. 13-20.

- Grandguillaume, Gilbert. «Langues et nation : le cas de l'Algérie ». Meynier, Gilbert : L'Algérie contemporaine. Bilan et solutions pour sortir de la crise. Paris :

L'Harmattan, Les Cahiers de Confluences, 2000. 89-99. Gilbert Grandguillaume. 29/06/2005 <http://grandguillaume.free.fr/ cont/Nancy.html>

- Grandguillaume, Gilbert. «Les enjeux de la question des langues en Algérie ». In : Bistolfi, Robert : Les Langues de la Méditerranée. Paris : L'Harmattan, Les Cahiers de Confluences, 2002. 141-165. Gilbert Grandguillaume. 29/06/2005 <http://grandguillaume.free.fr/cont/ enjlangues.html>

- Grandguillaume, Gilbert. «Les enjeux d'une politique linguistique ». In :

L'Interpénétration des cultures dans le Bassin occidental de la Méditerranée, Actes du Colloque de l'Association de la Méditerranée, Sorbonne, 14 novembre 2001. Paris : Mémoire de la Méditerranée, 2003. 99-110. Gilbert Grandguillaume. 29/06/2005 <http://grandguillaume.free.fr/cont/ intercultures.html> - Kaufmann, Jean-Claude. L'invention de soi. Une théorie de l'identité. Paris : Armand Colin, 2003.

- Lejeune, Philippe. Le Pacte autobiographique. Paris : Seuil, 1975.

- Liebreich, Sabine : Liberté et solitude dans les romans de Malika Mokeddem. Vienne :

Mémoire de Magister, sous la direction de Zohra Bouchentouf-Siagh, 2003 (avec une interview de Malika Mokeddem).

- Memmi, Albert. Portrait du colonisé. Portrait du colonisateur. Première publication : Corréa, 1957 ; Paris : Gallimard, 1985.

- Moati, Nine. Deux femmes à Paris. Première publication : Paris : Ramsay, 2000 ; Paris : Ramsay, 2007.

- Moati, Nine. Mon enfant, ma mère. Première publication : Paris : Stock, 1974 ; Paris : Ramsay, 2006.

- Mokeddem, Malika. Je dois tout à ton oubli. Paris : Grasset, 2008.

- Mokeddem, Malika. Mes Hommes. Paris : Grasset, 2005.

- Mokeddem, Malika. La Transe des insoumis. Paris : Grasset, 2003.

- Mokeddem, Malika. Le Siècle des sauterelles. Paris : Ramsay, 1992.

- Mokeddem, Malika. Les Hommes qui marchent. Paris : Ramsay, 1990.

Robin, Régine. Le Deuil de l'origine: une langue en trop, la langue en moins. Paris : Editions Kimé, 2003.

Sartre, Jean-Paul. Situations, V. Colonialisme et néo-colonialisme. Paris : Gallimard, 1964 Sebbar, Leïla. Voyage en Algéries autour de ma chambre. Saint-Pourçain-sur-Sioule : Bleu autour, 2008.

- Sebbar, Leïla. L'arabe comme un chant secret. Saint-Pourçain-sur-Sioule : Bleu autour, 2007

- Sebbar, Leïla. Journal de mes Algéries en France. Saint-Pourçain-sur-Sioule : Bleu autour, 2005 
- Sebbar, Leïla. Mes Algéries en France. Carnet de voyages. Préface de Michelle Perrot. Saint-Pourçain-sur-Sioule : Bleu autour, 2004

- Sebbar, Leïla. Je ne parle pas la langue de mon père. Paris : Julliard, 2003

- Sebbar, Leilla. Le silence des rives. Paris : Stock, 1993

Segarra, Marta. Leur pesant de poudre : romancières francophones du Maghreb. Paris :

L'Harmattan, 1997

Taleb-Ibrahimi, Khaoula. Les Algériens et leur(s) langue(s). Eléments pour une approche sociolinguistique de la société algérienne. Alger : Editions El Hikma, 1997. 